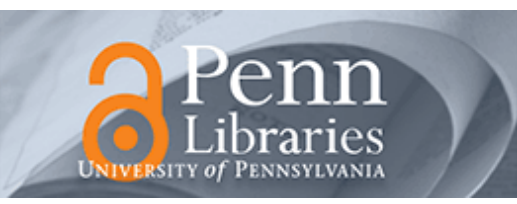

University of Pennsylvania

ScholarlyCommons

\title{
Demonstration of Low Voltage and Functionally Complete Logic Operations Using Body-Biased Complementary and Ultra-Thin ALN Piezoelectric Mechanical Switches
}

\author{
Nipun Sinha \\ University of Pennsylvania, nipun@seas.upenn.edu \\ Timothy Jones \\ University of Pennsylvania, jjonests@seas.upenn.edu \\ Zhijun Guo \\ University of Pennsylvania, zguo@seas.upenn.edu \\ Gianluca Piazza \\ Enlow this and additional works at: https://repository.upenn.edu/meam_papers \\ Part of the Mechanical Engineering Commons
}

\section{Recommended Citation}

Sinha, Nipun; Jones, Timothy; Guo, Zhijun; and Piazza, Gianluca, "Demonstration of Low Voltage and Functionally Complete Logic Operations Using Body-Biased Complementary and Ultra-Thin ALN Piezoelectric Mechanical Switches" (2010). Departmental Papers (MEAM). 258.

https://repository.upenn.edu/meam_papers/258

Suggested Citation:

Nipun Sinha, Timothy S. Jones, Zhijun Guo, and Gianluca Piazza. "DEMONSTRATION OF LOW VOLTAGE AND FUNCTIONALLY COMPLETE LOGIC OPERATIONS USING BODY-BIASED COMPLEMENTARY AND ULTRA-THIN ALN PIEZOELECTRIC MECHANICAL SWITCHES" Proceedings of 2010 MicroElectroMEchanical Systems Conference (MEMS 2010) (2010).

(C2010 IEEE. Personal use of this material is permitted. However, permission to reprint/republish this material for advertising or promotional purposes or for creating new collective works for resale or redistribution to servers or lists, or to reuse any copyrighted component of this work in other works must be obtained from the IEEE.

This paper is posted at ScholarlyCommons. https://repository.upenn.edu/meam_papers/258

For more information, please contact repository@pobox.upenn.edu. 


\title{
Demonstration of Low Voltage and Functionally Complete Logic Operations Using Body-Biased Complementary and Ultra-Thin ALN Piezoelectric Mechanical Switches
}

\author{
Abstract \\ This paper reports, for the first time, on the demonstration of low voltage and functionally complete logic \\ elements (NAND and NOR) implemented by using body-biased complementary and ultra-thin ( $250 \mathrm{~nm}$ \\ thick) Aluminum Nitride (AIN) based piezoelectric mechanical switches. This work presents, firstly, the \\ importance of scaling AIN films for the demonstration of ultra-thin AIN switches and, secondly, the \\ implementation of a new actuation scheme based on body biasing to lower the switch threshold voltage. \\ Four of these ultra-thin switches were connected together to synthesize functionally complete MEMS \\ logic gates (NAND and NOR) with $\mathrm{a} \pm 2 \mathrm{~V}$ swing and a body-bias voltage $<8 \mathrm{~V}$. \\ Disciplines \\ Engineering | Mechanical Engineering \\ Comments \\ Suggested Citation: \\ Nipun Sinha, Timothy S. Jones, Zhijun Guo, and Gianluca Piazza. "DEMONSTRATION OF LOW VOLTAGE \\ AND FUNCTIONALLY COMPLETE LOGIC OPERATIONS USING BODY-BIASED COMPLEMENTARY AND \\ ULTRA-THIN ALN PIEZOELECTRIC MECHANICAL SWITCHES" Proceedings of 2010 \\ MicroElectroMEchanical Systems Conference (MEMS 2010) (2010). \\ (C2010 IEEE. Personal use of this material is permitted. However, permission to reprint/republish this \\ material for advertising or promotional purposes or for creating new collective works for resale or \\ redistribution to servers or lists, or to reuse any copyrighted component of this work in other works must \\ be obtained from the IEEE.
}




\title{
DEMONSTRATION OF LOW VOLTAGE AND FUNCTIONALLY COMPLETE LOGIC OPERATIONS USING BODY-BIASED COMPLEMENTARY AND ULTRA-THIN ALN PIEZOELECTRIC MECHANICAL SWITCHES
}

\author{
Nipun Sinha, Timothy Jones, Zhijun Guo and Gianluca Piazza \\ University of Pennsylvania, USA
}

\begin{abstract}
This paper reports, for the first time, on the demonstration of low voltage and functionally complete logic elements (NAND and NOR) implemented by using body-biased complementary and ultra-thin $(250 \mathrm{~nm}$ thick) Aluminum Nitride (AlN) based piezoelectric mechanical switches. This work presents, firstly, the importance of scaling AlN films for the demonstration of ultra-thin AlN switches and, secondly, the implementation of a new actuation scheme based on body biasing to lower the switch threshold voltage. Four of these ultra-thin switches were connected together to synthesize functionally complete MEMS logic gates (NAND and NOR) with $\mathrm{a} \pm 2 \mathrm{~V}$ swing and a body-bias voltage $<8 \mathrm{~V}$.
\end{abstract}

\section{INTRODUCTION}

The CMOS industry has thrived thanks to the scaling of the transistor over the past few decades and has been able to implement smaller, faster and more energy efficient devices. Nonetheless, with the continuous scaling of the planar bulk metal-oxide semiconductor field effect transistors (MOSFETs) to the nanodimensions the International Technology Roadmap for Semiconductors (ITRS) [1] has recognized the emergence of some key issues in these extremely small devices, such as the variability and magnitude of the threshold voltages, the source-to-drain leakage in the standby state and the increased effect of parasitics like source/drain resistance due to the ultra-thin body of the device. For these reasons, the ITRS is also emphasizing the need to investigate alternate devices and technologies like NEMS switches that will lower transistor switching voltages and consume less power. These devices have the potential to replace the CMOS transistors in applications where low power consumption is of paramount importance.

Regular CMOS MOSFETs exhibit passive power dissipation due to the presence of a physical semiconductor channel between the source and the drain. The presence of this channel causes sub-threshold conduction and leads to standby power consumption.
The replacement of this channel with an air-gap would drastically reduce the leakage power consumption and lower the subthreshold slope well below the $60 \mathrm{mV} / \mathrm{dec}$ level of CMOS. Therefore, the presence of an air-gap simultaneously enables the reduction in leakage current and the implementation of a device with a threshold voltage of just few mVs. This new class of switches, based on an air-gap, can be considered mechanical transistors. MEMS switches [2] are readily available prototypes for verifying the characteristics of the mechanical transistor before scaling it into the NEMS regime. For this purpose, we have built an AlN piezoelectric MEMS switch [3]-[4]. Differently from all other actuation mechanisms like electrostatic [5]-[6], electromagnetic [7] and thermal [8], piezoelectric actuation gives us the advantage of being extremely linear in nature and requiring low power for actuation. Lead Zirconate Titanate (PZT) [9]-[10] and AlN [3]-[4] are the two most commonly used piezoelectric materials for realizing MEMS switches. Over PZT, AIN has the advantage of being CMOS compatible, exhibiting lower leakage current for comparable film thicknesses and having shown preservation of piezoelectric coefficients when scaled from bulk to ultra-thin films [11]. The scalability of AIN films makes it more amenable for NEMS switch development. In order to scale NEMS actuator, the two most important characteristics that need to be considered are deflections $(\delta)$ and resonance frequency $\left(f_{n}\right)$. Displacement, in fact, puts a constraint on the required switching voltage, achievable switching forces and manufacturability of the airgap. The frequency dictates the speed of operation of the transistor, which should be desirably scaled to a few nanoseconds. The relationship of deflection and resonance frequency to the various design parameters such as length $(L)$ and thickness $(t)$ are shown in equation (1).

$$
\delta \propto\left(\frac{L}{t}\right)^{2} \quad \text { and } \quad f_{n} \propto \frac{t}{L^{2}}
$$

The product of these two parameters shows that the key parameter that needs to be scaled in order to obtain high deflection and high resonance frequency is the film thickness, as shown in equation (2). 


$$
f_{n} \delta \propto \frac{1}{t}
$$

With the intent of moving towards nanoscaled devices, this work shows the first AlN piezoelectric switches developed by using $250 \mathrm{~nm}$ thick films. These films have shown a marked reduction in the actuation voltages of the switches and simultaneously permitted the demonstration, for the very first time, of low voltage $( \pm 2 \mathrm{~V}$ ) functionally complete (NAND and NOR) MEMS logic gates.

\section{PRINCIPLE OF OPERATION}

For demonstration of complementary logic using AlN MEMS switches a novel actuation methodology was used. In analogy to 4-terminal CMOS devices, the actuation scheme is called the body-biased actuation.

Piezoelectric actuation necessitates the presence of two actuation electrodes that sandwich the piezoelectric material and create an electric field across it, which generates the strain that leads to actuation. A body bias stands for a constant and floating voltage applied to one of the actuation electrodes. Conventionally, to turn the switch-on, one actuation electrode is shorted to ground and the other electrode has a potential greater than the threshold voltage. This mode of actuation can be referred to as the 'grounded actuation' mode. In the 'body-biased' mode of actuation the previously grounded actuation electrode is biased to a constant voltage (body-bias) very close in magnitude (but opposite in polarity) to the threshold voltage. In this scenario, actuation (closing and opening of the switch) can be achieved by applying a very small voltage on the other actuation electrode. Figure 1 schematically shows and compares the two actuation modes.

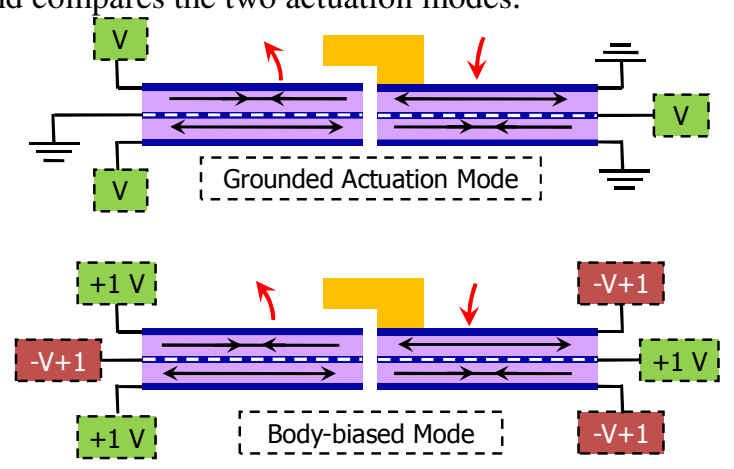

Fig. 1: Schematic representation of the principle of operation of an AlN switch in grounded and bodybiased mode of operation. By applying a bias to one of the metal layers $(-V+1 \mathrm{~V})$, the same effective electric field required to open and close the switch is applied across the AlN layer with just $1 \mathrm{~V}$. The gate voltage can be lowered to $<1 \mathrm{~V}(1 \mathrm{~V}$ is used in the figure $)$.

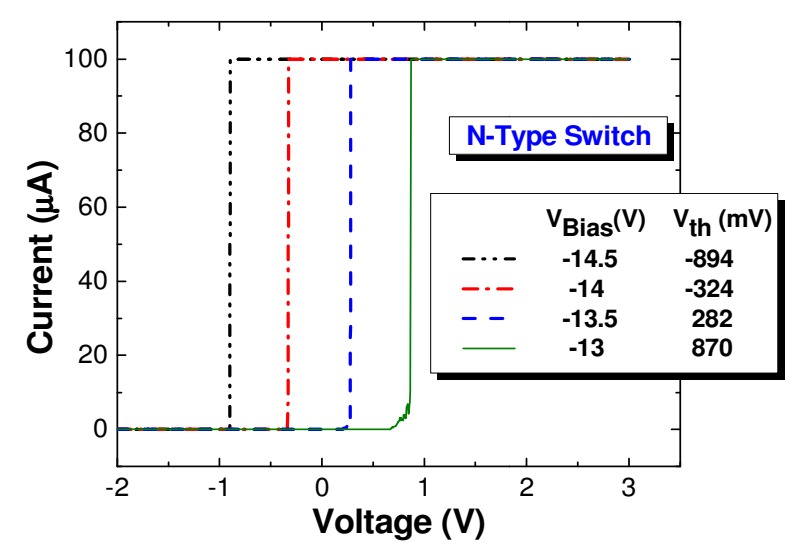

Fig. 2: Experimental data verifying that the switch threshold voltage $\left(V_{t h}\right)$ can be tuned by varying the value of the body bias. This data shows that the bodybias technique enables the implementation of variable threshold voltages on the same die and in the same process. The current measurement was limited to a maximum value of $100 \mu \mathrm{A}$.

Previously, threshold voltage manipulation [12] had been demonstrated in electrostatic switches by introducing a pre-charged electrode. In that case the precharging voltages were very high and there was a significant variation in threshold (pull-in) voltage with respect to time. Instead by using the body-biased threshold voltage control method, the threshold voltage of a piezoelectric switch can be linearly tailored to be any value, negative or positive, as shown in Figure 2.

The body-biasing technique also allows us to synthesize $\mathrm{n}$ or $\mathrm{p}$-type switches out of the same identical device without any changes in the design or fabrication. As expected, the devices also exhibit a very low subthreshold slope, measured to be $<1 \mathrm{mV} /$ dec.

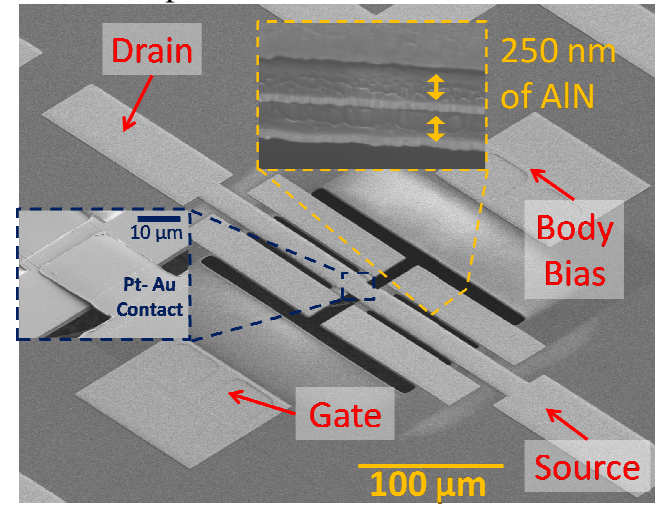

Fig. 3: SEM of an ultra-thin film dual-beam AlN switch illustrating the source, drain, gate and body bias terminals. One inset (top) shows the cross-sectional view of the actuator and the stacked AlN nano-films. The second inset (left) shows the flat tip and the nanogap in the switch. 
The major impact of the body-biasing technique is the ability to reduce the threshold voltage, which has been experimentally pushed to values as low as $10-30 \mathrm{mV}$ [13]. The scaling of the AlN films to $250 \mathrm{~nm}$ was introduced in order to reduce the required body-bias voltages and pave the way to ultimately scale the actuator dimensions to achieve fast switching at high forces. A reduction in the body bias, in fact, gives us more control over the desired threshold voltage. Figure 3 shows an SEM of an AIN MEMS switch with an inset showing the cross-section of the ultra-thin films that were used to make it. By leveraging on the scaling of the AlN films both the NAND and NOR gates were synthesized at low switching voltages $( \pm 2 \mathrm{~V})$ and with a body bias $<8 \mathrm{~V}$.

Despite the higher piezoelectric coefficient of PZT, previously shown PZT based NAND gates [14] required elevated voltages $(20 \mathrm{~V})$. Therefore, this thin-film AlN demonstration constitutes a significant step forward in the realization of low-voltage logic elements.

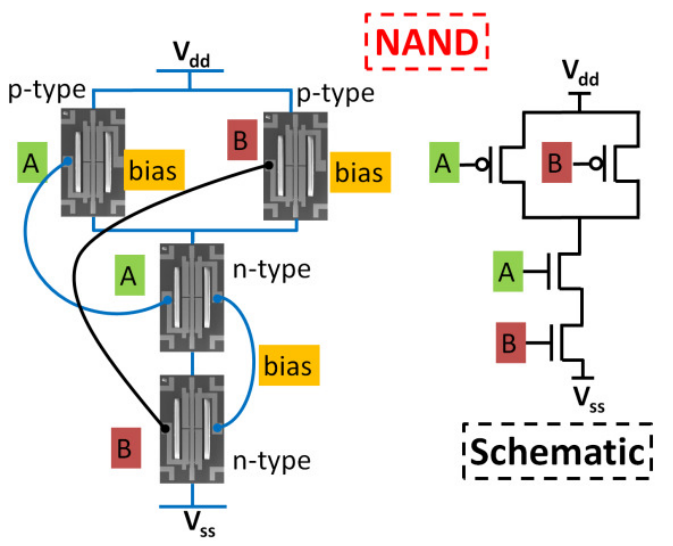

(a)

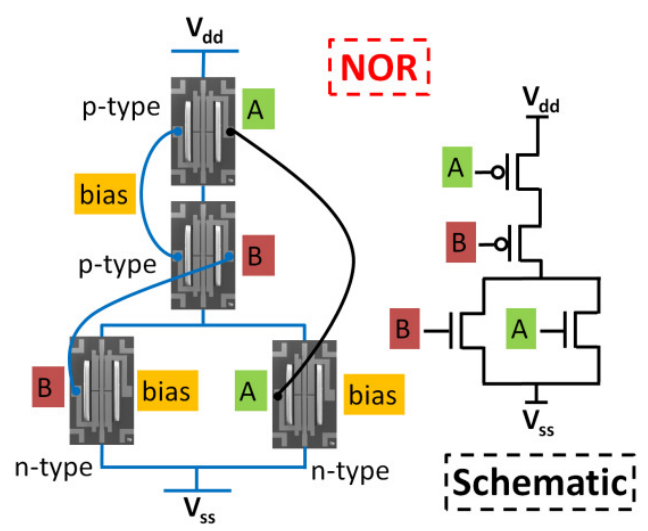

(b)

Fig 4: Layout showing the wiring of (a) the NAND and (b) the NOR for the testing of complementary logic and the electronic schematic of a conventional NAND and NOR, respectively.

\section{RESULTS}

Both the NAND and NOR structures were formed by wire-bonding four separate ultra-thin AlN switches in the desired configuration. Wire-bonding is used to prove the concept of using body-biasing for making complex logic elements in ultra-thin films. The same logic elements can be synthesized on chip via direct routing. No electrical amplifiers or buffers were used in the testing of these mechanical logic elements.

The graph in Figure 5 shows the results and equivalent truth table for the testing of a mechanical NAND formed by using four ultra-thin AlN mechanical switches (Fig. 4(a)) with one input at $100 \mathrm{~Hz}$ and the other at $50 \mathrm{~Hz}$ with a $\pm 2 \mathrm{~V}$ voltage swing. The frequencies of operation $(100 \mathrm{~Hz}$ and $50 \mathrm{~Hz})$ were selected for ease of operation and demonstration. The device is not limited to these frequencies and can potentially operate at higher frequencies. In fact, the rise time for these switches has been separately measured to be $<100$ nanoseconds. The body biases for this NAND structure were set at $+5.3 \mathrm{~V}$ for the two n-type switches and $-8 \mathrm{~V}$ and $-7.7 \mathrm{~V}$ for the $\mathrm{p}$-type switches.

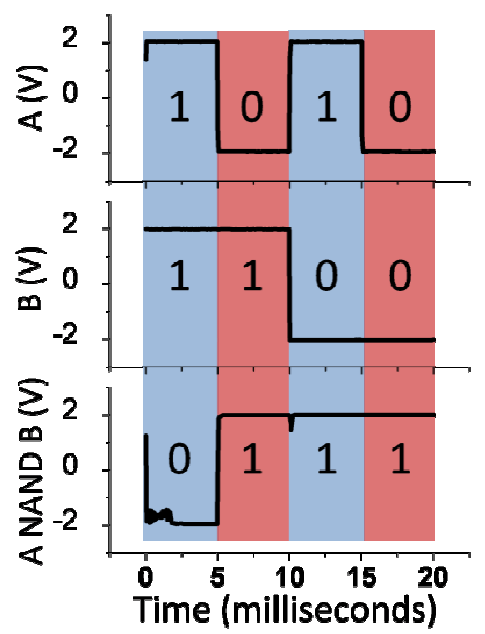

Fig. 5: Experimental data for one cycle of operation of a NAND gate made using ultra-thin AlN mechanical switches (250nm AlN layers) and its corresponding truth table. Note the $\pm 2 V$ swing.

The in plane dimensions of the switches have not been scaled proportionally to the thickness. Thus, the device stiffness is significantly reduced. This translates into mechanical bouncing when making contact. It is this dynamical instability that restricted the swing voltage to $\pm 2 \mathrm{~V}$. The bouncing is also the primary reason why the results in Figure 5 show some spikes in the reported data. The bouncing will be significantly attenuated when the structure is further miniaturized, since a higher equivalent stiffness can be engineered. Thus, overall 
scaling of the device dimensions will yield the ideal logic system, operating with switching voltages of just few mVs. The graph in Figure 6 shows the results and equivalent truth table for the testing of a mechanical NOR formed by four ultra-thin mechanical switches (Fig. 4(b)) with one input at $100 \mathrm{~Hz}$ and the other at $50 \mathrm{~Hz}$ with a $\pm 2 \mathrm{~V}$ voltage swing. The body biases for this NOR structure were set at $-4.5 \mathrm{~V}$ for the two p-type switches and $+6.7 \mathrm{~V}$ and $+5.5 \mathrm{~V}$ for the n-type switches. Preliminary contact reliability data for a $\mathrm{Au}$ to $\mathrm{Pt}$ contact shows that the resistance can be kept below $500 \Omega$ (sufficient for logic applications) for $>10^{7}$ cycles. This data hints that the main issues faced by RF MEMS switches might not be as problematic for computing applications.

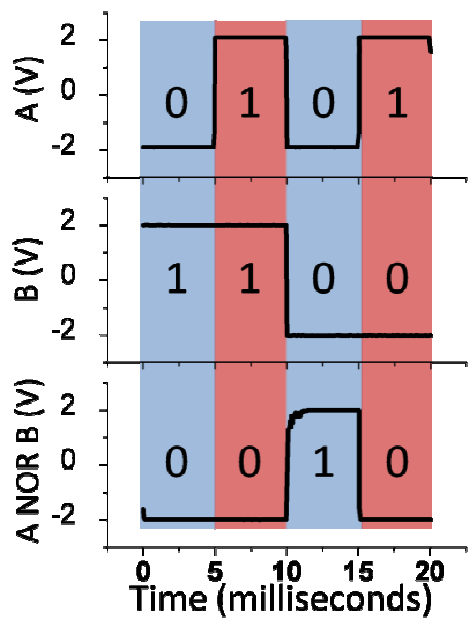

Fig. 6: Experimental data for one cycle of operation of a NOR gate made using ultra-thin AlN mechanical switches (250nm AlN layers) and its corresponding truth table. Note the $\pm 2 V$ swing.

\section{CONCLUSIONS}

This work demonstrates the scaling of AlN films to $250 \mathrm{~nm}$ for making body-biased NAND and NOR logic gates. The scaling is associated with lower threshold and body-bias voltages. NAND and NOR gates in fact operate with $\pm 2 \mathrm{~V}$ swing and $<8 \mathrm{~V}$ body bias. Further miniaturization of the switch to the nanoscale will lead to faster and ultra-low-voltage logic components.

\section{ACKNOWLEDGEMENTS}

This work was supported by the DARPA NEMS program. The authors would like to acknowledge the help of the staff of the Wolf Nanofabrication Facility at the University of Pennsylvania and the PRISM Micro/Nano Fabrication Laboratory at the Princeton University.

\section{REFERENCES}

[1] International Technology Roadmap for Semiconductors 2007 Edition, Austin, TX, Sematech.

[2] G. M. Rebeiz, "RF MEMS Theory, Design and Technology", $1^{\text {st }}$ Edition, John Wiley \& Sons, Inc., 2003.

[3] R. Mahamameed, N. Sinha, M. B. Pisani, and G. Piazza, "Dual Beam Actuation of Piezoelectric AlN RF MEMS Switches Monolithically Integrated with AIN Contourmode Resonators," Journal of Micromech. Microeng. 18, 105011, 2008.

[4] N. Sinha, R. Mahameed, C. Zuo, G. Piazza, "Integration of AlN micromechanical contour-mode technology filters with three-finger dual beam AIN MEMS switches," Frequency Control Symposium, 2009 Joint with the 22nd European Frequency and Time forum. IEEE International, pp.1-4, 20-24 April 2009.

[5] P. M. Zavracky, S. Majumder and N. E. McGruer, "Micromechanical switches fabricated using nickel surface micromachining," Jour. of MEMS, vol.6, no.1, pp.3-9, Mar 1997.

[6] C. Bozler, R. Drangmeister, S. Duffy, M. Gouker, J. Knecht, L. Kushner, R. Parr, S. Rabe, and L. Travis, "MEMS microswitch arrays for reconfigurable distributed microwave components", IEEE MTT-S International Microwave Symposium Digest, Boston, MA (June 2000), pp. 153-156.

[7] M. Ruan, J. Shen, and C. B. Wheeler, "Latching Micromagnetic Relays", Journal of MEMS, 10/2001, pp. 511-517.

[8] R. D. Streeter, C. A. Hall, R. Wood, and R. Madadevan, "VHF high-power tunable RF bandpass filter using microelectromechanical (MEM) microrelays", Int. J. RF Microwave CAE, 11/5/2001, pp. 261-275.

[9] H. C. Lee, J. H. Park, J. Y. Park, H. J. Nam and J. U. Bu "Design, fabrication and RF performances of two different types of piezoelectrically actuated Ohmic MEMS switches", Journal of Micromech. Microeng. , 15(2004), pp. 2098-2104.

[10] R. G. Polcawich, D. Judy, J. S. Pulskamp, S. TrolierMcKinstry and M. Dubey, "Advances in Piezoelectrically Actuated RF MEMS Switches and Phase Shifters", Microwave Symposium, (2007) IEEE/MTT-S International, pp.2083-2086.

[11] N. Sinha, G. E. Wabiszewski, R. Mahameed, V. V. Felmetsger, S. M. Tanner, R. W. Carpick, and G. Piazza, Appl. Phys. Lett. 95, 053106 (2009).

[12] H-H Yang, J. O. Lee and J-B Yoon, "Maneuvering Pullin Voltage of an Electrostatic Micro-switch by Introducing a Pre-charged Electrode," IEEE IEDM 2007, Washington DC, USA, pp. 439-442.

[13] N. Sinha, T. S. Jones, Z. Guo and G. Piazza, "BodyBiased Complementary Logic Implemented using AlN Piezoelectric MEMS Switches," to be presented at Intl. Electron Devices Meeting 2009.

[14] D. Judy, R. G. Polcawich, J. Pulskamp, "Low Voltage Complementary MEMS Logic using Piezoelectric Actuators," 2008 Solid State Sensor, Actuator and Microsystems Workshop, Hilton Head Island, South Carolina, pp. 328 - 331, 2008. 